\title{
Robust Precoding Design for Indoor MU-MISO Visible Light Communication
}

\author{
Houssem Sifaou, Abla Kammoun, Ki-Hong Park, Mohamed-Slim Alouini \\ Computer, Electrical, and Mathematical Sciences \& Engineering Division, \\ King Abdullah University of Science and Technology, Thuwal, Saudi Arabia
}

\begin{abstract}
Visible light communication (VLC) is recognized as a promising technology to complement existing wireless communication systems due to its main advantages such as ease of deployment, low cost and large unlicensed bandwidth. This paper considers the precoding design for a multi-user multiple-input-single-output VLC system. Two major concerns need to be considered while solving such a problem. The first one is related to the inter-user interference, basically inherent to our consideration of a multiuser system, while the second results from the users' mobility, causing imperfect channel estimates. To address both concerns, we propose robust precoding designs that solve both max-min SINR and minimal illumination level problems. The first problem allows users' rates maximization while ensuring fairness and the second problem decides the feasibility of a certain set of target SINRs. The proposed robust designs are studied under different conditions, and are shown to achieve a high gain over their nonrobust counterparts.
\end{abstract}

Index Terms-Visible light communication, multi-user multipleinput-single-output system, robust precoding design, max-min SINR, minimal illumination level.

\section{INTRODUCTION}

Due to the increasing demand for high data rate, radio frequency (RF) communication is now facing a serious spectrum shortage. One of the promising technologies that can be deployed to complement RF communication is optical wireless communication (OWC), and particularly visible light communication (VLC) for indoor environments. VLC is an emerging technology that employs light-emitting diodes (LEDs) that, aside from their initial role as illuminating devices, are used for communication purposes [1], [2]. In the long run, these LEDs are expected to substitute conventional lighting devices used for indoor illumination due to their reliability, low cost, and high power efficiency. High data rate transmission can be performed using LEDs by modulating the light intensity, which, at the receiver, is converted into an electric signal by one or multiple receiving photo-detectors (PDs). Such light intensity fluctuations are too fast to be detected by the human eye, which allows to not disrupt the role of LEDs as illuminating devices.

The prospect of enhancing system performance through using multi-user multiple-input-single-output (MU-MISO) techniques for VLC systems is now gaining an increasing interest among researchers in optical wireless communications [3]-[6]. This becomes all the more natural that many transmitters are usually used for illumination. The same transmitters can be thus used to transmit downlink data streams to many user equipments (UEs). This, however, comes along with many several technical challenges that have to be addressed, among which is the mitigation of inter-user interference, naturally arising in multiuser systems. Such a problem has been addressed in RF communication by employing precoding schemes, [7]-[12]. These techniques cannot be directly implemented to VLC systems because of many technical considerations in VLC. These considerations encompass, for instance, the positivity of the signal and the necessity of operating in the linear dynamic range of the LEDs [3]-[6]. As a consequence, a considerable effort has recently been devoted to propose precoding schemes adapted to the practical conditions of VLC systems. In this regard, several precoding techniques built upon different design criteria have been proposed. Particularly, in [4] precoding schemes that minimize the mean square error (MSE) have been proposed while the maximization of the minimum signal-to-interferenceplus-noise (SINR) design objective has been considered in [3], [6].

All these works, however, have relied on the assumption of perfect knowledge of the channel state information. While such an assumption is usually considered in indoor VLC systems in case of static UEs, it fails to hold when the mobility of the UEs is considered. To overcome this issue, we consider in this paper the design of robust precoding schemes taking into account imperfect channel state information (CSI) estimate due to mobility of the UEs. Based on an outdated CSI uncertainty model, we propose two robust precoding schemes addressing the maxmin SINR problem and the minimization of the illumination level under some target SINR constraints. The first problem allows UEs' rates maximization while ensuring fairness and the second problem decides the feasibility of a set of target SINRs required by the UEs and minimize the power needed to satisfy these SINR constraints in case of feasibility. To the best of our knowledge, the only work addressing the design of robust precoding from sum MSE minimization and minimal illumination level perspectives for VLC systems is due to [5]. However, unlike our work, the precoding in [5] is conceived to satisfy target MSE constraints. As SINR constraints are directly related to the throughput, they are more pertinent, and as such our design might be more relevant in practice than that in [5].

The remainder of this paper is organized as follows. The next section describes the system model. In section III, robust precoding schemes are proposed for both max-min SINR and minimal illumination level problems. In section IV, the performance of the proposed schemes is discussed using numerical simulations and section $\mathrm{V}$ concludes the paper. 


\section{SySTEM MOdeL}

We consider a multi-user MISO VLC system where $M$ LED transmitters communicate with $K$ UEs. Each UE is equipped with a single receiving PD. At the LED transmitters, the input electric signal is converted into light intensity, and at the receiver, the PD converts the light intensity into electric signal. To comply with some technical considerations of VLC systems, three constraints on the input electric signal have to be accounted for. First, in order to satisfy a certain illumination level, the expectation of the input electric signal $y_{n}$ at every transmitter should be equal to a certain value $I_{D C}^{n}: \mathbb{E}\left(y_{n}\right)=I_{D C}^{n}$. Second, it is natural to impose the same brightness level at every LED transmitter, which formally implies that: $I_{D C}^{n}=I_{D C}, \forall n$. This may be possible for instance by deploying LED transmitters uniformly on the roof, thus allowing equal illumination level over the room area. Finally, it is important to ensure that each LED operates in its linear dynamic range [4] by requiring the input electric signal to satisfy:

$$
I_{L} \leq y_{n} \leq I_{U}
$$

where $I_{U}>I_{L}>0$ represent the upper and lower bounds of the LED drive current in the linear range [4]. For complexity concerns, we assume that the transmitters, which are fully coordinated, employ linear precoding. Let $s_{k} \in\{-1,1\}$ be the symbol intended to UE $k$, assumed to be drawn from a BPSK constellation. Denote by $\mathbf{W}=\left[w_{n, k}\right] \in \mathbb{R}^{M \times K}$ the precoding matrix. The transmitted signal at the $n$-th LED transmitter becomes:

$$
x_{n}=\sum_{k=1}^{K} s_{k} w_{n, k} .
$$

In order to satisfy the constraint $\mathbb{E}\left(y_{n}\right)=I_{D C}, \quad \forall n$, a DC offset $I_{D C}$ should be added to $x_{n}, y_{n}=x_{n}+I_{D C}$. While to satisfy $I_{L} \leq y_{n} \leq I_{U}$, the precoding matrix $\mathbf{W}$ should be conceived so that:

$$
\sum_{k=1}^{K}\left|w_{n, k}\right| \leq, I_{\min } \quad n=1, \cdots, M .
$$

where $I_{\min }=\min \left(I_{D C}-I_{L}, I_{U}-I_{D C}\right)$. In indoor VLC systems, the line of sight (LOS) propagation prevails [13], [14]. Thus, the channel gain between the $n$-th transmitter and the $k$-th receiver is often modeled as [13], [15]:

$$
h_{n, k}=\left\{\begin{array}{rlrl}
\frac{\rho c A}{d_{n, k}^{2}} R\left(\phi_{n, k}\right) \cos \left(\theta_{n, k}\right), & & \theta_{n, k} \leq \theta_{c} \\
0, & \theta_{n, k}>\theta_{c}
\end{array}\right.
$$

where $\phi_{n, k}$ is the angle of emission with respect to the $n$-th transmitter, $\theta_{n, k}$ is the incident angle with respect to the $k$ th receiver, $d_{n, k}$ is the distance between the $n$-th transmitter and the $k$-th receiver, $\theta_{c}$ is the receiver filed of view (FOV), $\rho$ is the PD responsivity, $c$ is the LED conversion factor and $A=\frac{q^{2}}{\sin ^{2}\left(\theta_{c}\right)} A_{P D}$ is the collection area, with $q$ is the refractive index of optical concentrator and $A_{P D}$ is the PD area. $R\left(\phi_{n, k}\right)$ is the Lambertian radiant intensity defined as:

$$
R\left(\phi_{n, k}\right)=\frac{(m+1) \cos ^{m}\left(\phi_{n, k}\right)}{2 \pi},
$$

where $m$ is the order of the Lambertian emission mode number [13], [15]. For notation convenience, we define the channel vector corresponding to the $k$-th UE as $\mathbf{h}_{k}=\left[h_{1, k}, \cdots, h_{M, k}\right]^{T}$. At the receiver, the contribution of the DC offset $I_{D C}$, introduced at the transmitter, in the received signal is removed. The useful received signal at the $k$-th UE is thus given by:

$$
r_{k}=\mathbf{h}_{k}^{T} \mathbf{w}_{k} s_{k}+\sum_{j \neq k} \mathbf{h}_{k}^{T} \mathbf{w}_{j} s_{j}+z_{k},
$$

where $z_{k}$ is the additive noise. For VLC systems, $z_{k}$ is modeled as a real valued Gaussian random variable with zero mean and variance $\sigma_{k}^{2}$ given by [14]:

$$
\sigma_{k}^{2}=2 e B\left(I_{s, k}+I_{b g} I_{2}\right)+B i_{a m p}^{2},
$$

where $e$ is the electronic charge, $B$ is the system bandwidth, $I_{b g}$ is the background current, $I_{2}$ is the noise bandwidth factor, $i_{a m p}$ is the preamplifier noise density and $I_{s, k}$ is defined as:

$$
I_{s, k}=\sum_{n=1}^{M} I_{D C} h_{n, k} .
$$

The vector $\mathbf{r}$ collecting the received signals at the UEs can be expressed as: $\mathbf{r}=\mathbf{H W} \mathbf{s}+\mathbf{z}$, where $\mathbf{H} \in \mathbb{R}^{K \times M}$ is the channel matrix, $\mathbf{s}=\left[s_{1}, \cdots, s_{K}\right]^{T}$ denotes the symbol vector and $\mathbf{z}=$ $\left[z_{1}, \cdots, z_{K}\right]^{T}$ is the noise vector. The signal-to-interferenceplus-noise ratio (SINR) at the $k$-th UE can be expressed as:

$$
\operatorname{SINR}_{k}=\frac{\left|\mathbf{h}_{k}^{T} \mathbf{w}_{k}\right|^{2}}{\sum_{j \neq k}\left|\mathbf{h}_{k}^{T} \mathbf{w}_{j}\right|^{2}+\sigma_{k}^{2}} .
$$

A considerable effort has been devoted to the task of designing the optimal matrix $\mathbf{W}$ that solves a given optimization problem involving the SINR. Most of the proposed designs assume perfect knowledge of the channel, which is a valid assumption in VLC as long as the users are static. This, however, no longer holds when users are mobile, which might cause the performance to be far below the expectations. To overcome this issue, we propose in the next section linear precoding schemes that are more robust to imperfect knowledge of the channel.

\section{Robust Precoding Design}

In this section, we propose robust precoding techniques based on two different design criteria: max-min SINR and minimization of the illumination level. Prior to presenting our designs, we shall first introduce the channel uncertainty model adopted in this work.

\section{A. Uncertainty model}

In this paper, we assume an outdated CSI model [5], [16]. The true channel vector $\mathbf{h}_{k}$ is expressed as the sum of the channel vector estimate $\widehat{\mathbf{h}}_{k}$ and an error vector $\boldsymbol{\epsilon}_{k}$ that represents CSI uncertainty:

$$
\mathbf{h}_{k}=\widehat{\mathbf{h}}_{k}+\boldsymbol{\epsilon}_{k},
$$

We assume a bounded uncertainty model, in which $\epsilon_{k}$ is assumed to satisfy: $\left\|\boldsymbol{\epsilon}_{k}\right\|_{2} \leq \delta_{k}$, where $\delta_{k}$ is an error bound assumed to be known at the transmit side. Such a model is suitable to account for the mobility of the UEs in VLC systems 


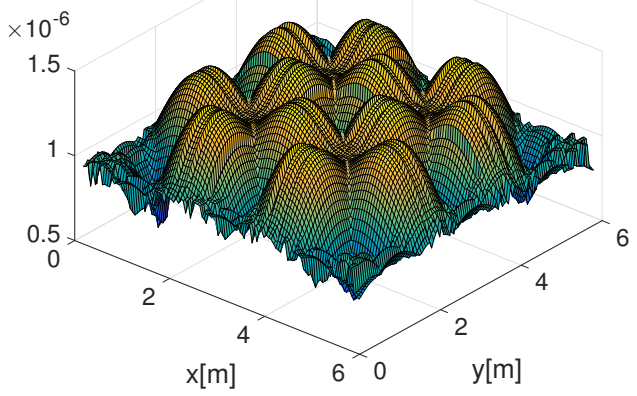

Fig. 1: Error bounds obtained by simulation for $L=0.25$. VLC system settings used are described in section IV.

where the channel error results from the discrepancy in the actual UE position. To determine $\delta_{k}$, we assume that between two CSI updates, the UE may travel a maximal distance $L$ from the initial UE position $\mathbf{p}_{1}$. The uncertainty region is thus represented by the disk of radius $L$ and center $\mathbf{p}_{1}$. With these assumptions at hand, $\delta_{k}$ associated with position $\mathbf{p}_{1}$ is computed by generating many candidate UE positions uniformly at random in the uncertainty region and taking the maximum norm of the obtained error vectors. This results in the following uncertainty region:

$$
\mathcal{U}\left(\delta_{k}\right)=\left\{\mathbf{h}_{k} \mid \mathbf{h}_{k}=\widehat{\mathbf{h}}_{k}+\boldsymbol{\epsilon}_{k},\left\|\boldsymbol{\epsilon}_{k}\right\|_{2} \leq \delta_{k}\right\} .
$$

For the sake of illustration, we represent in Fig. 1 the error bounds $\delta_{k}$ across the room area for $L=0.25$.

\section{B. Robust design for max-min SINR problem}

In this section, we propose a robust precoding design that solves the max-min SINR problem. Prior to that, we find it instructive to review the max-min SINR problem in the perfect CSI case, solved in our work [6]. The max-min SINR problem in the perfect CSI case can be written as:

$$
\begin{aligned}
& \mathcal{P}: \max _{\mathbf{W}} \min _{k} \frac{\mathrm{SINR}_{k}}{\gamma_{k}} \\
& \text { C: } \sum_{k=1}^{K}\left|w_{n, k}\right| \leq I_{\min }, \forall n
\end{aligned}
$$

where $\left\{\gamma_{k}\right\}$ are scalars reflecting the priority given to the UEs. Problem $(\mathcal{P})$ can be rewritten as:

$$
\begin{aligned}
\mathcal{P}_{1}: & \max _{\mathbf{W}, t} t \\
& \mathbf{C}_{1}: \sum_{k=1}^{K}\left|w_{n, k}\right| \leq I_{\text {min }}, \quad \forall n, \\
& \mathrm{C}_{2}: \operatorname{SINR}_{k} \geq \gamma_{k} t, \quad \forall k,
\end{aligned}
$$

If $t$ is fixed, constraints $\mathrm{C}_{1}$ and $\mathrm{C}_{2}$ define a convex set. Problem $\mathcal{P}$ can be thus solved using a bisection search method. Starting from a feasible value $t_{1}$ and an unfeasible $t_{2}$, the bisection algorithm looks for the largest feasible $t$ for which there exists a precoding matrix $\mathbf{W}$ that satisfies $\mathrm{C}_{1}$ and $\mathrm{C}_{2}$. This amounts to solving, at every iteration, the following second-order cone programming (SOCP) feasibility problem

$$
\begin{aligned}
& \text { find } \mathbf{w} \\
& \mathbf{C}_{1}:\left\|\mathbf{B}_{k} \mathbf{w}+\boldsymbol{\sigma}_{k}\right\|_{2} \leq \frac{\mathbf{h}_{k}^{T}\left(\mathbf{I}_{M} \otimes \mathbf{e}_{k}^{T}\right) \mathbf{w}}{\sqrt{\gamma_{k} t}}, \quad \forall k, \\
& \mathbf{C}_{2}:-\mathbf{a} \leq \mathbf{w} \leq \mathbf{a}, \quad \mathbf{C}_{3}: \mathbf{U a} \leq I_{\min } \mathbf{1}_{M}
\end{aligned}
$$

with $\mathbf{B}_{k} \in \mathbb{R}^{(K+1) \times M K}$ and $\boldsymbol{\sigma}_{k} \in \mathbb{R}^{(K+1)}$ are given by:

$$
\mathbf{B}_{k}=\left[\begin{array}{l}
\mathbf{h}_{k}^{T} \otimes \mathbf{I}_{K}^{k} \\
\mathbf{0}_{1 \times M K}
\end{array}\right], \quad \boldsymbol{\sigma}_{k}=\left[0, \cdots, 0, \sigma_{k}\right]^{T},
$$

where $\mathbf{I}_{K}^{k}$ denotes the matrix obtained from identity matrix of size $K$ by setting the $(k, k)$-th element to zero, $\mathbf{e}_{k}$ is the all zeros vector having the $k$-th element equal to $1, \otimes$ is the Kronecker product and $\mathbf{U}=\mathbf{I}_{M} \otimes \mathbf{1}_{K}^{T} . \mathbf{a} \in \mathbb{R}^{M K \times 1}$ is a slack variable and $\mathbf{w} \in \mathbb{R}^{M K \times 1}$ is defined as $\mathbf{w}=\operatorname{vec}\left(\mathbf{W}^{T}\right)=$ $\left[\mathbf{e}_{1}^{T} \mathbf{W}, \cdots, \mathbf{e}_{M}^{T} \mathbf{W}\right]^{T}$. More details on this reformulation can be found in [6]. For the sake of completeness, we outline the steps of the bisection algorithm solving $\mathcal{P}_{1}$ :

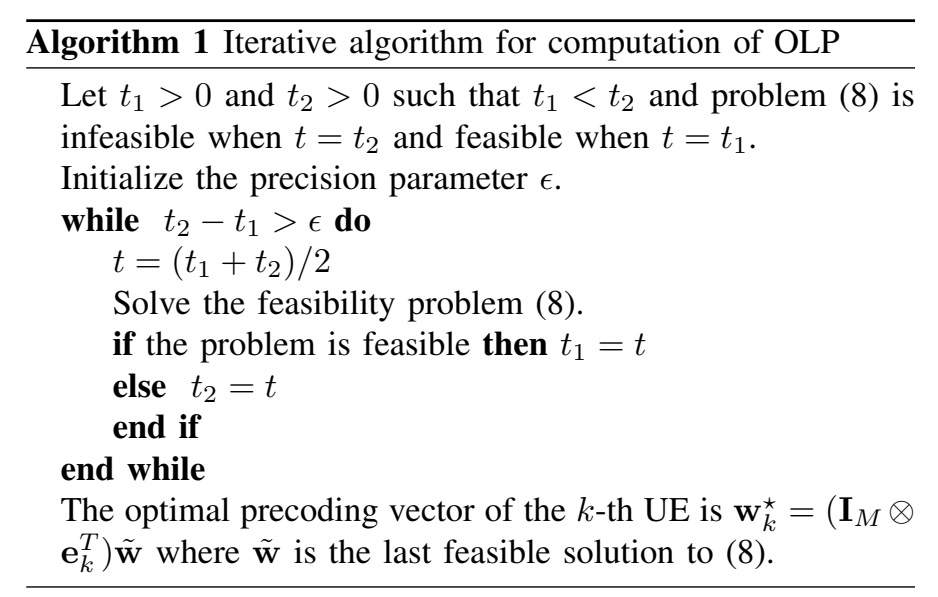

In the imperfect CSI case, the max-min SINR problem can be solved using a similar approach. The main difference with the perfect CSI case lies in the SINR constraints which must be satisfied for any channel vector $\mathbf{h}_{k}$ in the uncertainty region $\mathcal{U}\left(\delta_{k}\right)$, resulting into the following optimization problem:

$$
\begin{aligned}
\mathcal{P}_{r}: & \max _{\mathbf{W}, t} t \\
& \mathrm{C}_{1}: \sum_{k=1}^{K}\left|w_{n, k}\right| \leq I_{\min }, \forall n \\
& \mathrm{C}_{2}: \operatorname{SINR}_{k} \geq \gamma_{k} t, \quad \forall \mathbf{h}_{k} \in \mathcal{U}\left(\delta_{k}\right), \forall k
\end{aligned}
$$

Problem $\mathcal{P}_{r}$ can be solved using an iterative bisection search method aiming to determine the largest value of $t$ for which it exists a precoding matrix solving the following feasibility problem:

$$
\begin{aligned}
& \text { find } \mathbf{w} \\
& \mathrm{C}_{1}:\left\|\mathbf{B}_{k} \mathbf{w}+\boldsymbol{\sigma}_{k}\right\|_{2} \leq \frac{\mathbf{h}_{k}^{T}\left(\mathbf{I}_{M} \otimes \mathbf{e}_{k}^{T}\right) \mathbf{w}}{\sqrt{\gamma_{k} t}}, \quad \mathbf{h}_{k} \in \mathcal{U}\left(\delta_{k}\right), \forall k, \\
& \mathrm{C}_{2}:-\mathbf{a} \leq \mathbf{w} \leq \mathbf{a}, \quad \mathrm{C}_{3}: \mathbf{U} \leq I_{\min } \mathbf{1}_{M},
\end{aligned}
$$


The above feasibility problem is no longer an SOCP problem as in the perfect CSI case. To solve it, we should resort to techniques from robust convex optimization [17]. Applying the results of [17], the $K$ second-order cone constraints $C_{1}$ in problem (9) are equivalent to the following $2 K$ semi-definite programming (SDP) constraints:

$$
\boldsymbol{\Phi}_{k} \succeq 0, \quad \mathbf{\Psi}_{k} \succeq 0, \quad \forall k=1, \cdots, K
$$

where

$$
\boldsymbol{\Phi}_{k}=\left[\begin{array}{cccc}
\frac{\widehat{\mathbf{h}}_{k}^{T} \mathbf{w}_{k}}{\sqrt{\gamma_{k} t}-\lambda_{k}} & \frac{\delta_{k} w_{k, 1}}{\sqrt{\gamma_{k} t}} & \ldots & \frac{\delta_{k} w_{k, M}}{\sqrt{\gamma_{k} t}} \\
\frac{\delta_{k} w_{k, 1}}{\sqrt{\gamma_{k} t}} & \frac{\widehat{\mathbf{h}}_{k}^{T} \mathbf{w}_{k}}{\sqrt{\gamma_{k} t}}-\lambda_{k} & \ldots & 0 \\
\vdots & \vdots & \ddots & \vdots \\
\frac{\delta_{k} w_{k, M}}{\sqrt{\gamma_{k} t}} & 0 & \ldots & \frac{\widehat{\mathbf{h}}_{k}^{T} \mathbf{w}_{k}}{\sqrt{\gamma_{k} t}}-\lambda_{k}
\end{array}\right],
$$

and

$$
\mathbf{\Psi}_{k}=\left[\begin{array}{ccccc}
\lambda_{k}-\mu_{k} & 0 & \ldots & 0 & {\left[\mathbf{A}_{k}^{0} \mathbf{w}+\boldsymbol{\sigma}_{k}\right]^{T}} \\
0 & \mu_{k} & & & {\left[\mathbf{A}_{k}^{1} \mathbf{w}\right]^{T}} \\
\vdots & & \ddots & & \vdots \\
0 & & & \mu_{k} & {\left[\mathbf{A}_{k}^{M} \mathbf{w}\right]^{T}} \\
\mathbf{A}_{k}^{0} \mathbf{w}+\boldsymbol{\sigma}_{\boldsymbol{k}} & \mathbf{A}_{k}^{1} \mathbf{w} & \ldots & \mathbf{A}_{k}^{M} \mathbf{w} & \lambda_{k} \mathbf{I}
\end{array}\right]
$$

with $\left\{\lambda_{k}\right\}$ and $\left\{\mu_{k}\right\}$ are newly introduced optimization variables and $\mathbf{A}_{k}^{j} \in \mathbb{R}^{(K+1) \times M K}$ are defined as:

$$
\mathbf{A}_{k}^{0}=\left[\begin{array}{l}
\widehat{\mathbf{h}}_{k}^{T} \otimes \mathbf{I}_{K}^{k} \\
\mathbf{0}_{1 \times M K}
\end{array}\right], \quad \mathbf{A}_{k}^{j}=\left[\begin{array}{l}
\delta_{k} \mathbf{e}_{j}^{T} \otimes \mathbf{I}_{K}^{k} \\
\mathbf{0}_{1 \times M K}
\end{array}\right], \quad 1 \leq j \leq M .
$$

Thus, the feasibility problem in (9) can be reformulated as:

$$
\begin{aligned}
& \text { find } \quad \mathbf{w} \\
& \mathrm{C}_{1}: \quad \boldsymbol{\Phi}_{k} \succeq 0, \quad \forall k, \quad \mathrm{C}_{2}: \quad \boldsymbol{\Psi}_{k} \succeq 0, \quad \forall k, \\
& \mathrm{C}_{3}: \quad-\mathbf{a} \leq \mathbf{w} \leq \mathbf{a}, \quad \mathrm{C}_{4}: \quad \mathbf{U a} \leq I_{\min } \mathbf{1}_{M},
\end{aligned}
$$

which turns out to be an SDP problem that can be efficiently solved using CVX [18].

\section{Robust design for minimal illumination problem}

In this section, for the sake of tractability, we assume that the noise variance is independent of $I_{D C}$ and we use an upper bound of the noise variance while solving this optimization problem (i. e., $\sigma_{k}^{2}$ is computed for the maximal value of $I_{D C}$ which is $\left.\left(I_{U}+I_{L}\right) / 2\right)$. However, true noise variance is used in the numerical simulation. In the perfect CSI case, the minimal illumination problem can be written as follows:

$$
\begin{aligned}
\mathcal{P}_{2}: & \min _{\mathbf{W}, I_{D C}} I_{D C} \\
& \mathrm{C}_{1}: \operatorname{SINR}_{\mathrm{k}} \geq \gamma_{k}, \forall k \\
& \mathrm{C}_{2}: \sum_{k=1}^{K}\left|w_{n, k}\right| \leq I_{\min }, \forall n
\end{aligned}
$$

where $\left\{\gamma_{k}\right\}$ are a set of target SINRs. This problem can be equivalently written as:

$$
\begin{aligned}
\mathcal{P}_{3}: & \min _{\mathbf{W}, I_{D C}} I_{D C} \\
& \mathrm{C}_{1}:\left\|\mathbf{B}_{k} \mathbf{w}+\boldsymbol{\sigma}_{k}\right\|_{2} \leq \frac{\mathbf{h}_{k}^{T}}{\sqrt{\gamma_{k}}}\left(\mathbf{I}_{M} \otimes \mathbf{e}_{k}^{T}\right) \mathbf{w}, \quad \forall k, \\
& \mathrm{C}_{2}:-\mathbf{a} \leq \mathbf{w} \leq \mathbf{a}, \quad \mathbf{C}_{3}: \mathbf{U} \leq I_{\min } \mathbf{1}_{M},
\end{aligned}
$$

where the quantities $\mathbf{B}_{k}, \boldsymbol{\sigma}_{k}$, w and $\mathbf{a}$ are the same quantities defined in the previous section. This is an SOCP problem that can be efficiently solved using CVX [18]. For the imperfect CSI case, the minimal illumination problem is modified as:

$$
\begin{aligned}
& \mathcal{P}_{4}: \min _{\mathbf{W}, I_{D C}} I_{D C} \\
& \mathrm{C}_{1}:\left\|\mathbf{B}_{k} \mathbf{w}+\boldsymbol{\sigma}_{k}\right\|_{2} \leq \frac{\mathbf{h}_{k}^{T}\left(\mathbf{I}_{M} \otimes \mathbf{e}_{k}^{T}\right) \mathbf{w}}{\sqrt{\gamma_{k}}}, \mathbf{h}_{k} \in \mathcal{U}\left(\delta_{k}\right), \forall k \\
& \mathrm{C}_{2}:-\mathbf{a} \leq \mathbf{w} \leq \mathbf{a} \\
& \mathrm{C}_{3}: \mathbf{U} \mathbf{a} \leq I_{\min } \mathbf{1}_{M}
\end{aligned}
$$

This problem can be solved using the same approach as in the previous section. In fact, using again results from [17], it can be shown that it is equivalent to the following SDP problem:

$$
\begin{aligned}
& \mathcal{P}_{5}: \min _{\mathbf{W}, I_{D C}} I_{D C} \\
& \mathrm{C}_{1}: \quad \tilde{\boldsymbol{\Phi}}_{k} \succeq 0, \quad \forall k, \quad \mathrm{C}_{2}: \mathbf{\Psi}_{k} \succeq 0, \quad \forall k, \\
& \mathrm{C}_{3}: \quad-\mathbf{a} \leq \mathbf{w} \leq \mathbf{a}, \quad \mathbf{C}_{4}: \quad \mathbf{U a} \leq I_{\min } \mathbf{1}_{M},
\end{aligned}
$$

where $\tilde{\boldsymbol{\Phi}}_{k}$ are the matrices obtained from $\boldsymbol{\Phi}_{k}$ by setting $t$ to 1 . In case of feasibility, $\mathcal{P}_{5}$ can be efficiently solved using CVX [18].

\section{NUMERICAL RESULTS}

In this section, the performance of the proposed precoding design is studied using numerical simulations. We consider a VLC system composed of nine LED arrays installed on the ceiling of the room at height $z=3$. Each LED array is composed of 36 LEDs and is viewed as a single transmitter. The positions of the LED arrays are described in Fig. 2. In all simulations, 4 UEs are used and their positions are chosen randomly in the plane at height $z=0.8$. The UEs coordinates take thus the form $(x, y, 0.8)$ for some random $x$ and $y$. In all Monte Carlo simulations, we average over 5000 channel realizations.Table 1 is presented to help the reader keep track of the different system parameters.

\section{A. Minimum SINR maximization problem}

We begin by investigating the performance of the proposed max-min SINR robust precoding. To this end, we start from the assumed UEs locations from which we obtain the channel vector estimate $\widehat{\mathbf{h}}_{k}$ of UE $k$. In this part, the UEs priorities $\left\{\gamma_{k}\right\}$ are randomly chosen in the interval [1,2]. Then, given a distance bound $L$, the corresponding uncertainty error bound $\delta_{k}$ is computed as explained in section III-A. With $\widehat{\mathbf{h}}_{k}$ and $\delta_{k}$ on hand, the precoding matrix $\mathbf{W}$ is obtained by solving problem $\mathcal{P}_{r}$ through a bisection iterative approach. To assess 
TABLE 1: VLC system parameters.

\begin{tabular}{|l|l|}
\hline Room Size $\ell \times w \times h$ & $6 \mathrm{~m} \times 6 \mathrm{~m} \times 3 \mathrm{~m}$ \\
\hline LED dimensions $\ell \times w \times h$ & $3 \mathrm{~cm} \times 3 \mathrm{~cm} \times 2 \mathrm{~cm}$ \\
\hline LED spacing & $1 \mathrm{~cm}$ \\
\hline$I_{L}$ & $400 \mathrm{~mA}$ \\
\hline$I_{U}$ & $600 \mathrm{~mA}$ \\
\hline Mode number $m$ of Lambertian emission & 1 \\
\hline Photo Detector reponsivity $\rho$ & $0.4 \mathrm{~A} / \mathrm{W}$ \\
\hline Photo Detector area $A_{P D}$ & $1 \mathrm{~cm}^{2}$ \\
\hline Receiver FOV $\theta_{c}$ & $60 \mathrm{deg}$ \\
\hline Refractive index of optical concentrator $q$ & 1.5 \\
\hline Pre-amplifier noise density $i_{a m p}$ & $5 \mathrm{pA} / \mathrm{Hz}^{-1 / 2}$ \\
\hline Background current $I_{b g}$ & $100 \mu \mathrm{A}$ \\
\hline Noise bandwidth factor $I_{2}$ & 0.562 \\
\hline LED conversion factor $c$ & $0.44 \mathrm{~W} / \mathrm{A}$ \\
\hline Bandwidth $B$ & $10 \mathrm{MHz}$ \\
\hline
\end{tabular}

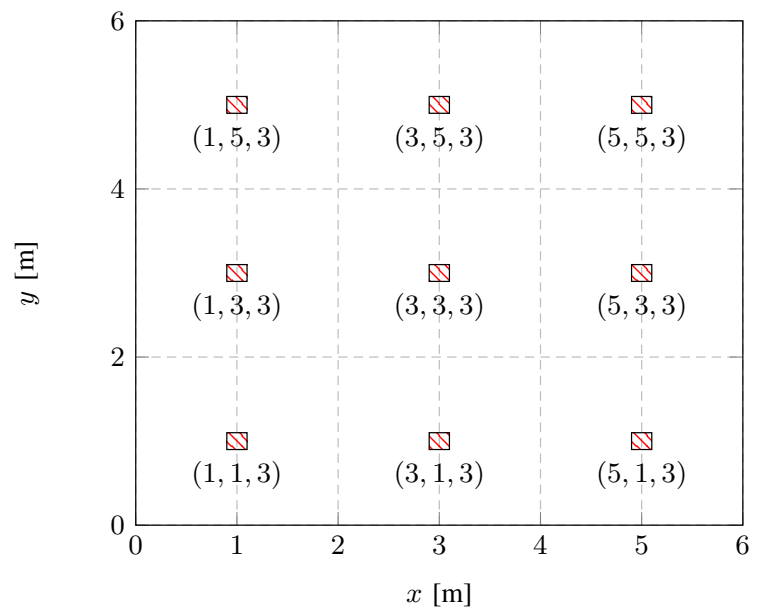

Fig. 2: Positions of the LED arrays on the roof.

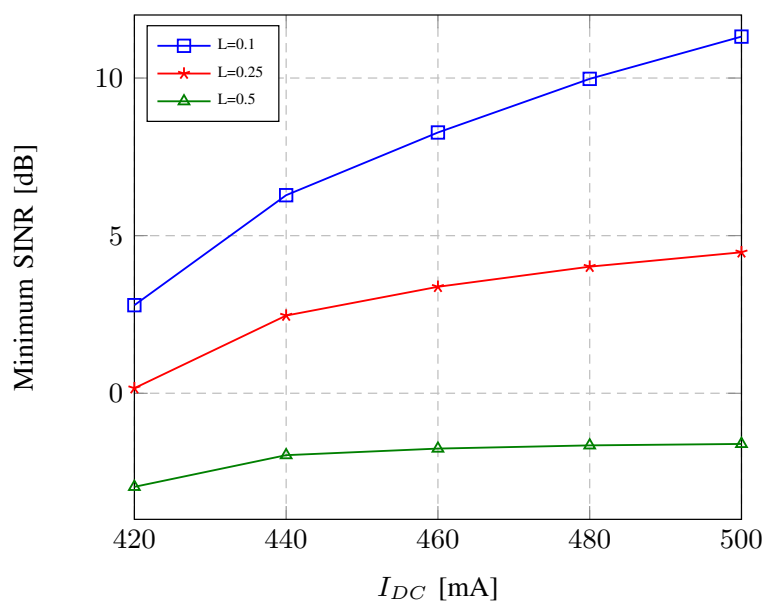

Fig. 3: Minimum SINR vs. DC offset $I_{D C}$ for $L=0.1,0.25,0.5$. Max min SINR problem with robust design.

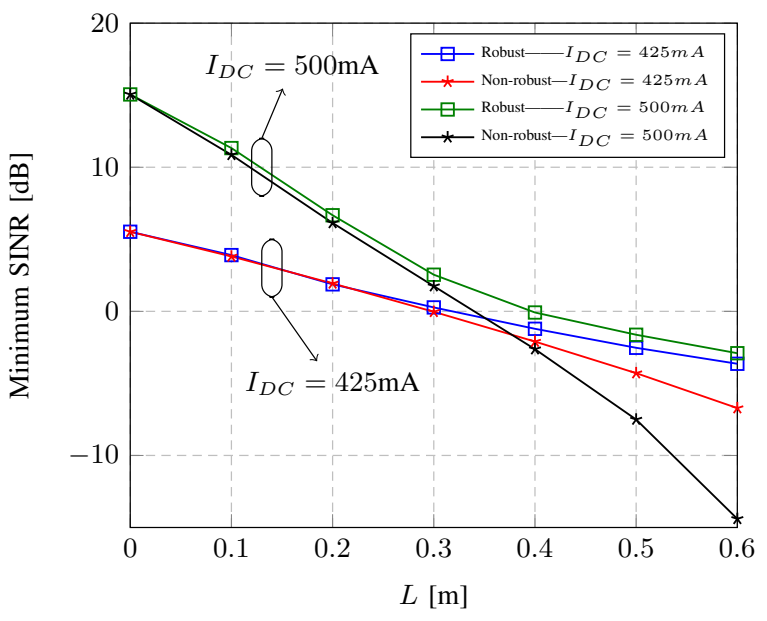

Fig. 4: Minimum SINR vs. distance bound $L$. Comparison between robust and non-robust design for max-min SINR problem.

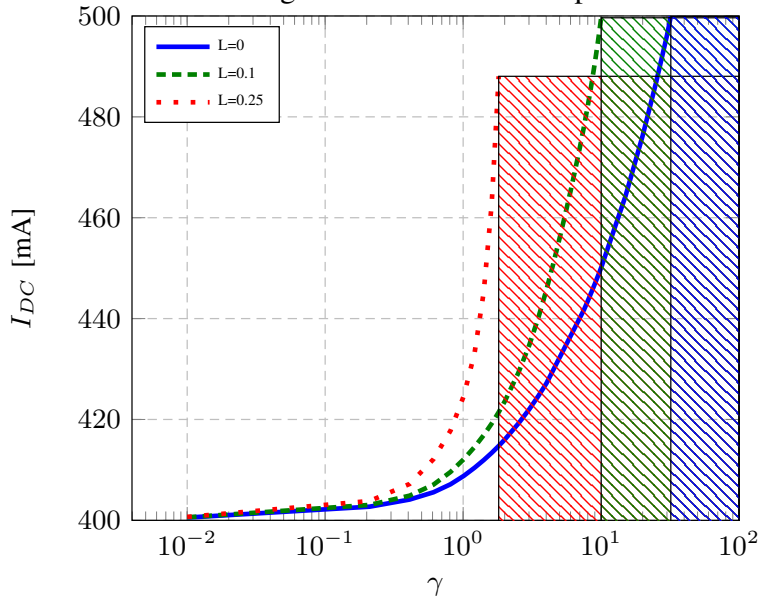

Fig. 5: DC offset $I_{D C}$ vs. SINR constraint $\gamma$. Minimal illumination level problem with robust design.

the performance of the proposed robust design, true channel gain vectors $\mathbf{h}_{k}$ representing the actual channel vector of UE $k$ and their corresponding SINRs are obtained by placing many UEs uniformly at random into the corresponding uncertainty region, which is defined as the disc of radius $L$ centered at the UE position during channel estimation. The performance of the proposed robust design is assessed in terms of the minimum SINR over these realizations of $\mathbf{h}_{k}$.

Fig. 3 reports the performance of the proposed robust precoding that solves the max-min SINR problem. In particular, we plot the minimum SINR vs. the DC offset $I_{D C}$ for different values of the distance bound $L$. As expected, the performance of the proposed design decreases with the distance bound $L$ and increases with the DC offset $I_{D C}$ up to the value $\left(I_{L}+I_{U}\right) / 2$. However, if $I_{D C}$ exceeds $\left(I_{L}+I_{U}\right) / 2$, lower performances are obtained. This principally comes from the per-transmitter constraint $\sum_{k=1}^{n}\left|w_{n, k}\right| \leq \min \left(I_{D C}-I_{L}, I_{U}-I_{D C}\right)$, which becomes the less stringent when $I_{D C}=\left(I_{L}+I_{U}\right) / 2$. Over the range $\left(I_{D C}=\left(I_{L}+I_{U}\right) / 2, I_{U}-I_{D C}\right)$, which is in passing not represented in Fig. 3, the average SINR curve will decrease, presenting a symmetric curve with respect to the vertical axis 
$I_{D C}=\left(I_{L}+I_{U}\right) / 2$.

As a second investigation, we study the advantages of the robust design over the non-robust one that wrongly assumes that $\widehat{\mathbf{h}}_{k}$ coincides with the actual channel vector. The non-robust design is obtained by solving problem $P_{1}$ in which $\widehat{\mathbf{h}}_{k}$ is used in place of $\mathbf{h}_{k}$ in the expression of the SINR. Fig. 4 plots the minimum SINR vs. the distance bound $L$ for both robust and nonrobust designs. As seen, the robust design clearly outperforms the non-robust one with a gap that gets larger as $L$ increases. Moreover, it is worth mentioning that above a certain value of $L$, the non-robust design performance does not necessarily improve when $I_{D C}$ increases in the range $\left[0,\left(I_{L}+I_{U}\right) / 2\right]$. Thus, increasing $I_{D C}$ results in an important energy-efficiency loss when $L$ is large. This must be compared with the robustdesign, the performance of which, even though approaching a certain limit value for large $L$, is always increasing with $I_{D C}$ in the range $\left(0,\left(I_{L}+I_{U}\right) / 2\right)$. As the gain becomes small with $L$ sufficiently large, we deduce that increasing the power may not be an energy efficient option as it does not lead to a significant gain in performance.

\section{B. Minimal illumination level problem}

The robust precoding design based on minimizing the illumination level over target SINRs constraints is now studied. For simulation purposes and without loss of generality, we assume in this part that the target SINRs $\gamma_{k}$ are the same for all UEs, i. e. $\gamma_{k}=\gamma, \quad \forall k$. We plot in Fig. 5 the minimal illumination level vs. target SINR $\gamma$ for different values of the distance bound $L$. As expected, the required illumination level increases with $L$ and with the SINR target $\gamma$. Moreover, for slow values of the target SINR, the increase with respect to $\gamma$ is linear, while it becomes exponential as we approach boundaries of the feasibility regions. Finally, it is worth pointing out that the robust design for the minimal illumination problem ensures by construction the SINR constraints, while the nonrobust design would likely fail to achieve it, as it would be based on mismatched values of the SINR computed using the wrongly estimated channels of the UEs.

\section{CONClusion}

In this work, we have proposed robust precoding designs for MU-MISO VLC derived using a channel uncertainty model accounting for the mobility of UEs. Two design criteria have been considered, namely the maximization of the minimum SINR and the minimization of the illumination level. Both problems have major practical significance, the former maximizes the UEs' rates while ensuring fairness, while the latter aims to design, in case of feasibility, the precoding that yields the minimal illumination level while satisfying a set of target SINR constraints required by the UEs. Numerical simulations have been presented to illustrate the gain of the robust design over the non-robust one. More investigations can be found in the journal version of this paper [19] where the MU-MIMO case has been studied and the joint robust design of the precoding and receiver has been considered.

\section{REFERENCES}

[1] D. Tsonev, H. Chun, S. Rajbhandari, J. J. D. McKendry, S. Videv, E. Gu, M. Haji, S. Watson, A. E. Kelly, G. Faulkner, M. D. Dawson, H. Haas, and D. O'Brien, "A 3-Gb/s single-LED OFDM-based wireless VLC link using a Gallium Nitride $\mu$ LED," IEEE Photonics Technology Letters, vol. 26, no. 7, pp. 637-640, Apr. 2014.

[2] D. Tsonev, S. Videv, and H. Haas, "Towards a $100 \mathrm{~Gb} / \mathrm{s}$ visible light wireless access network," Opt. Express, vol. 23, no. 2, pp. 1627-1637, Jan. 2015.

[3] Z. Yu, R. J. Baxley, and G. T. Zhou, "Multi-user MISO broadcasting for indoor visible light communication," in IEEE International Conference on Acoustics, Speech and Signal Processing, Vancouver, Canada, May 2013, pp. 4849-4853.

[4] B. Li, J. Wang, R. Zhang, H. Shen, C. Zhao, and L. Hanzo, "Multiuser MISO transceiver design for indoor downlink visible light communication under per-LED optical power constraints," IEEE Photonics Journal, vol. 7, no. 4, pp. 1-15, Aug. 2015.

[5] H. Ma, L. Lampe, and S. Hranilovic, "Coordinated broadcasting for multiuser indoor visible light communication systems," IEEE Transactions on Communications, vol. 63, no. 9, pp. 3313-3324, Sept. 2015.

[6] H. Sifaou, K.-H. Park, A. Kammoun, and M.-S. Alouini, "Optimal linear precoding for indoor visible light communication system," in Proc. IEEE ICC, Paris, France, May 2017.

[7] D. W. H. Cai, T. Q. S. Quekand, C. Wei Tan, and S. H. Low, "Maxmin SINR coordinated multipoint downlink transmission - duality and algorithms," IEEE Transactions on Signal Processing, vol. 60, pp. 53845395, 2012.

[8] H. Sifaou, A. Kammoun, L. Sanguinetti, M. Debbah, and M.-S. Alouini, "Max-min SINR low complexity transceiver design for single cell massive MIMO," in 2016 IEEE 17th International Workshop on Signal Processing Advances in Wireless Communications (SPAWC), Edinburgh, UK, Jul. 2016, pp. 1-6.

[9] H. Sifaou, A. Kammoun, L. Sanguinetti, M. Debbah, and M.-S. Alouini, "Max-min SINR in large-scale single-cell MU-MIMO: Asymptotic analysis and low complexity transceivers," IEEE Trans. Signal Processing, vol. 65, no. 7, pp. 1841-1854, Apr. 2017.

[10] K. Cumanan, L. Musavian, S. Lambotharan, and A. B. Gershman, "SINR balancing technique for downlink beamforming in cognitive radio networks," IEEE Signal Processing Letters, vol. 17, no. 2, pp. 133-136, Feb. 2010.

[11] H. Shen, Y. Deng, W. Xu, and C. Zhao, "Rate-maximized zero-forcing beamforming for vlc multiuser miso downlinks," IEEE Photonics Journal, vol. 8, no. 1, pp. 1-13, Feb. 2016.

[12] H. Shen, Y. Deng, W. Xu, and C. Zhao, "Rate maximization for downlink multiuser visible light communications," IEEE Access, vol. 4, pp. 65676573, 2016.

[13] L. Zeng, D. C. O’Brien, H. L. Minh, G. E. Faulkner, K. Lee, D. Jung, Y. Oh, and E. T. Won, "High data rate multiple input multiple output (MIMO) optical wireless communications using white led lighting," IEEE Journal on Selected Areas in Communications, vol. 27, no. 9, pp. 16541662, Dec. 2009.

[14] T. Komine and M. Nakagawa, "Fundamental analysis for visiblelight communication system using LED lights," IEEE Transactions on Consumer Electronics, vol. 50, no. 1, pp. 100-107, Feb. 2004.

[15] J. M. Khan and J. R. Barry, "Wireless infrared communications," Proceedings of the IEEE, vol. 85, no. 2, pp. 265-298, 1997.

[16] M. B. Shenouda and T. N. Davidson, "On the design of linear transceivers for multiuser systems with channel uncertainty," IEEE Journal on Selected Areas in Communications, vol. 26, no. 6, pp. 1015-1024, Aug. 2008.

[17] A. Ben-Tal and A. Nemirovski, "Robust convex optimization," Math. Oper. Res., vol. 23, no. 4, pp. 769-805, 1998.

[18] Inc. CVX Research, "CVX: Matlab software for disciplined convex programming, version 2.0 beta," Sept. 2012.

[19] H. Sifaou, A. Kammoun, K. H. Park, and M. S. Alouini, "Robust transceivers design for multi-stream multi-user mimo visible light communication," IEEE Access, Dec. 2017. 